\title{
Qingdao dans l'imaginaire colonial allemand du premier vingtième siècle
}

\section{Clémence Andreys}

\section{CpenEdition}

\section{Journals}

Édition électronique

URL : http://journals.openedition.org/ifha/466

DOI : $10.4000 /$ ifha. 466

ISSN : 2198-8943

\section{Éditeur}

IFRA - Institut franco-allemand (sciences historiques et sociales)

\section{Édition imprimée}

Date de publication : 30 septembre 2012

Pagination : 214-217

ISSN : 2190-0078

\section{Référence électronique}

Clémence Andreys, « Qingdao dans l'imaginaire colonial allemand du premier vingtième siècle », Revue de l'IFHA [En ligne], 4 | 2012, mis en ligne le 14 février 2013, consulté le 01 mai 2019. URL : http:// journals.openedition.org/ifha/466 ; DOI : 10.4000/ifha.466

Ce document a été généré automatiquement le 1 mai 2019.

(CIFHA 


\title{
Qingdao dans l'imaginaire colonial allemand du premier vingtième siècle
}

\author{
Clémence Andreys
}

Si l'aventure coloniale allemande est encore mal connue du grand public en Allemagne, force est de constater le regain d'intérêt des chercheurs pour ce thème depuis ces vingt dernières années, notamment sous l'influence des études postcoloniales. L'accent a été mis sur la culture coloniale, c'est-à-dire sur l'impact de la colonisation dans la métropole. Il est important de souligner que la place des colonies dans l'esprit et le cœur de la société allemande n'était pas aussi minime qu'on a pu le penser sans que l'on puisse pour autant les mettre sur le même plan que les colonies françaises et britanniques. En France, la présence allemande en Chine reste un terrain de recherche encore peu exploré. Cette étude souhaite donc proposer un point de vue extérieur à ce chapitre de l'histoire des relations sino-allemandes et donner à un public français le moyen d'appréhender la question du colonialisme allemand en Chine.

Elle s'inscrit dans une démarche qui privilégie les questions sociales et culturelles en visant une exploration de l'imaginaire colonial allemand - de sa genèse à la constitution d'une mémoire coloniale - à travers le cas de Qingdao. Il s'agit de décrypter les constructions et les stratégies discursives qui ont précédé, accompagné et suivi la colonisation et de déconstruire la mise en scène de la «colonie modèle ». Ce travail permet de réexaminer la façon dont s'est exprimé l'impérialisme allemand à travers l'exemple de Qingdao et d'évaluer la diffusion d'une conscience coloniale ainsi que la perception qu'a eue la population allemande de cette action. Revenir sur la colonisation, c'est aussi réfléchir à l'intime intrication du colonial avec l'histoire nationale, car l'expansion coloniale est liée à une volonté d'affirmation nationale. L'analyse des images apporte un éclairage inédit sur le processus de définition d'une identité collective allemande au tournant du siècle.

A la croisée des études germaniques et de l'histoire de la Chine, ce travail interdisciplinaire conjugue différentes approches : une approche culturaliste qui tente de comprendre et de déconstruire les représentations actives dans la culture allemande, une 
approche visuelle dans le sens où l'image est un élément essentiel de la diffusion de l'idéologie coloniale et une approche postcoloniale qui montre l'impact du projet dans la métropole et refuse une lecture dichotomique des rapports entre les communautés en mettant au centre les notions d'échange et d'interculturalité.

L'originalité de ce projet est notamment d'utiliser des sources très variées et, pour certaines, peu valorisées dans les recherches sur le sujet. Ce sont des sources officielles et des sources privées, des sources allemandes et, dans une moindre mesure, des sources chinoises, des sources textuelles et des sources visuelles comme des photographies, des cartes postales, des articles de presse à travers un panorama associant journaux nationaux et régionaux, journaux publiés en Chine et journaux publiés en Allemagne, discours au Reichstag, mémorandums du gouvernement, journaux intimes, correspondances, mémoires, fictions, etc. Le croisement de ces sources a permis de mettre en évidence des regards différents sur Qingdao, leur évolution et d'observer la constitution d'une mémoire coloniale.

La première partie expose les mécanismes de l'expansion allemande ultramarine tout en s'attachant aux questions particulières que posa la conquête de Qingdao pour pouvoir mieux appréhender le décodage du discours. Sont particulièrement mises en relief l'entreprise de colonisation des esprits et la place de Qingdao dans la sphère publique.

La deuxième partie s'articule autour des concepts du Soi et de l'Autre et vise à décrypter différents regards du Soi sur l'Autre pour mieux comprendre la construction de l'identité wilhelminienne. L'instrumentalisation des images par les acteurs de la colonisation, la mise en scène de l'altérité coloniale, à la fois objet d'exotisme, de mépris et d'idéalisation, les pratiques et les enjeux qui s'y rapportent sont aussi examinés.

La question du rapport entre la métropole et la périphérie et celle du mimétisme colonial sont au cœur de la troisième partie. Une germanisation du territoire à bail fut mise en œuvre par l'appropriation de l'espace chinois et l'établissement de pratiques sociales visant la stricte séparation des deux communautés et la création d'une "colonie modèle $"$.

Dans un ultime développement, il est question de la dynamique mémorielle, de la façon dont les individus et les groupes ont inscrit leurs souvenirs de cette période dans l'espace privé et dans l'espace public. Qingdao devient, dans cette dernière partie, un objet de mémoire. Les ressorts de la mythification et de la mystification sont particulièrement mis en relief.

Le croisement des différentes échelles - locale, nationale et internationale - en jeu dans la construction de l'image de Qingdao, font apparaître en quoi cet événement qu'est la colonisation de Qingdao s'insère dans "l'histoire globale» tout en soulignant ses spécificités.

La particularité de la présence allemande dans le Shandong tient d'abord au fait qu'une seule puissance occidentale occupa un territoire donné et tenta une expérience originale. Il faut en effet rappeler la dimension étatique du projet et le dirigisme avec lequel il fut mis en place. L'administration du territoire par la Marine a aussi impliqué un lien particulier entre le discours sur la colonie et la propagande pour la flotte militaire de l'empire. Enfin, la volonté de construire un établissement modèle pour les Chinois, les autres puissances et l'Allemagne wilhelminienne est tout à fait originale. Il s'agissait de confirmer au reste du monde que l'Allemagne avait la capacité et les atouts d'une puissance coloniale. Pour autant, les colonisateurs allemands comprirent petit à petit 
qu'ils ne pouvaient pas négliger l'environnement chinois. Ils prirent rapidement conscience qu'une politique autoritaire ne menait qu'à des conflits comme celui des Boxeurs et ne ferait pas progresser les intérêts économiques allemands en Chine; ils misèrent alors sur la politique culturelle pour le développement de Qingdao.

Si l'on essaie de définir les différences entre les protectorats africains et celui de Qingdao, on remarque aussi que le traitement iconographique est spécifique : si l'on retrouve les figures « imposées » de l'imagerie coloniale, il se dégage des clichés plus une tentative de rapprochement avec l'Autre, un effort de compréhension, un intérêt, qu'une réification de l'Autre par des mesures et des clichés anthropométriques qui ont pour but de le classifier. De même, les photographies des femmes chinoises sont peu communes hors du cercle familial et sont rarement là pour nourrir les fantasmes masculins occidentaux.

Enfin, on constate que Qingdao, tout comme les territoires allemands dans le Pacifique, a peu à peu perdu de son importance aux yeux des dirigeants, qui n'ont plus misé que sur la valorisation des territoires africains, tout en devenant un lieu de mémoire pour des individus et des groupes.

Travailler sur le discours colonial, c'est dévoiler un mécanisme de communication et de persuasion collective et analyser en même temps les limites entre la réalité et le mythe, entre l'événement historique et sa perception, l'histoire et la mémoire. L'œuvre accomplie à Qingdao oscille entre une mise en scène de l'identité nationale sous le signe de la tradition et la volonté d'expérimenter des projets dans la colonie qui pourraient être adaptés par la suite à la métropole. Cette étude permet de mettre en évidence une transformation du rapport à Qingdao dans la mentalité allemande. Elle permet de la mesurer et de comprendre dans quelle mesure les représentations impériales étaient encore influentes dans la métropole après l'ère coloniale. Cette période postérieure à l'occupation allemande est aussi intéressante du point de vue social : une analyse de la communauté allemande à partir des années 1920-1930 et jusqu'en 1949 pourrait être menée pour prolonger ce travail.

\section{AUTEUR}

\section{CLÉMENCE ANDREYS}

Clémence Andréys a présenté et soutenu sa thèse de doctorat le 5 décembre 2011 à l'université Louis Lumière-Lyon II sous la direction de Jacques Poumet. 